\title{
MULTI-WAVELENGTH OBSERVATIONS OF NOVAE IN OUTBURST
}

\author{
S. Starrfield \\ IGPP and Theoretical Division, Los Alamos National Laboratory \\ and Department of Physics and Astronomy, Arizona State University
}

\section{INTRODUCTION}

A nova outburst is the second most violent explosion that can occur in a galaxy. While less violent than a supernova explosion, novae are considerably more frequent. More than 250 have been observed in the last century and there were more than a dozen novae being observed in outburst when this review was written. Over the past few years they have been studied at wavelengths ranging from the radio to the X-ray and these data have provided exciting new information about the nova outburst. For example, IUE studies have shown that there are two classes of novae; those that occur on carbon-oxygen white dwarfs and those that occur on oxygen-neon-magnesium white dwarfs. EXOSAT observations have shown that an intense X-ray emitting source exists in the system for years after the initial outburst has been discovered and radio studies have found that some novae are ejecting material in jets. IR studies have found high excitation coronal lines, dust formation, and have provided accurate distances. Optical studies have provided determinations of densities and temperatures in the ejecta and have discovered a class of novae that occur on strongly magnetized white dwarfs. There have been predictions but no detections of $\gamma$-ray emission. Finally, novae are time dependant and simultaneous or contemporaneous observations are required in order to learn as much as possible about the characteristics of the outburst.

Novae are members of the general class of close binary systems referred to as cataclysmic variables. A cataclysmic variable (CV) contains a Roche Lobe filling secondary, on or near the main sequence, losing hydrogen-rich material through the inner Lagrangian point onto an accretion disk that surrounds a white dwarf. Neither the physical processes that drive the secondary into overfilling its Roche Lobe are known, nor are the processes that remove angular momentum from the gas and allow it to spiral onto the white dwarf. Nevertheless, the existence of the nova outburst is sufficient to demonstrate that material is flowing onto the white dwarf.

The accreted layer grows in thickness until it reaches a temperature that is high enough for the initiation of thermonuclear burning of hydrogen in the accreted envelope. The consequences of the ensuing thermonuclear runaway (hereafter TNR) will depend upon the mass and luminosity of the white dwarf, the rate of mass accretion, and the chemical composition of the reacting layer. The numerical simulations of this evolution resemble the observations of the nova outburst (see Truran 1982; Starrfield 1986, 1988, 1989; Shara 1989 for reviews). The observed abundances of the intermediate mass elements in the ejecta demonstrate that accretion onto the white dwarf can dredge-up material from the core, so that the novae outburst is actually sampling the interior of the white dwarf (Truran and Livio 1986; Sparks et al. 1988). Therefore, by studying the ejecta from a variety of novae, we can gain important information about the evolutionary history of the white dwarfs prior to the classical nova phase of their evolution. Finally, 
since they are dredging up core material that contains intermediate mass elements and processing it through a hot hydrogen burning region, novae can be expected to eject material that is rich in the odd isotopes of abundant elements and they may be the galactic source of these species.

Since a nova explosion involves the ejection of more than $\sim 10^{-5} M_{\odot}$ to $\sim 10^{-4} M_{\odot}$ into space at velocities exceeding hundreds of kilometers per second and it lasts only a few years, one can study gas dynamics on short time scales as compared to most astronomical objects. IR observations of novae in outburst demonstrate that they are one of the astrophysical sites where grains are found and there is strong observational evidence for grain formation and growth in nova ejecta. Other time dependent phenomena observed in novae include nebular line emission and stellar winds.

This review serves as the introduction to the observational studies of novae and I will mention a number of results that will be emphasized by other reviewers. Therefore, I will try to provide the physical framework for multi-wavelength observations as applied to studies of novae. I divide the outburst into phases based on the physical effects that are occurring at that time. The first phase is the rise to bolometric maximum and occurs on a convective time scale. The second phase is the rise to visual maximum and occurs on the time scale for the envelope to expand to $\sim 10^{12} \mathrm{~cm}$. The third phase is the time when the nova is emitting at constant bolometric luminosity, but declining optical magnitude, and it lasts until most of the accreted material has been either exhausted or eroded from the surface of the white dwarf. The fourth and final phase is the return to quiescence (turn-off phase) and it occurs at the time that nuclear burning is ending. I will discuss each of these phases in turn and end with a discussion.

\section{PHASE I: THE RISE TO BOLOMETRIC MAXIMUM}

A nova outburst is classified, according to the rate of decline from maximum, either as "fast" or "slow." Many features of the outburst are correlated with speed class such as peak $\mathrm{M}_{B o l}$, ejection velocity, amount of material ejected during the early stages of the outburst, and chemical composition of the ejecta. It has also been found that the fastest novae are so violent that their maximum luminosities exceed $\mathrm{L}_{E d d}$. The explanation for this phenomenon is that at maximum the envelopes of novae are completely convective and the time scale is sufficiently short for convection to transport the $\beta^{+}$-unstable nuclei to the surface. In addition, at maximum the $\beta^{+}$-unstable nuclei are the most abundant of the CNO nuclei in the envelope, so that the rate of nuclear energy generation at the surface can grow to values exceeding $10^{12}$ to $10^{14} \mathrm{erg} \mathrm{gm}^{-1} \mathrm{sec}^{-1}$. This high a rate of energy generation at the surface can produce super- Eddington luminosities.

One of the most important results of the numerical simulations has been the identification of the role played by the $\beta^{+}$-unstable nuclei in the outburst. As the temperatures in the shell source exceed $\sim 10^{8} \mathrm{~K}$, the abundances of these nuclei $\left({ }^{13} \mathrm{~N},{ }^{14} \mathrm{O},{ }^{15} \mathrm{O}\right.$, and ${ }^{17} \mathrm{~F}$ ) increase to values where they severely impact the nuclear energy generation since every proton capture must now be followed by a waiting period before the $\beta^{+}$decay occurs and the daughter nucleus captures a proton. This has several effects on the subsequent evolution. First, since the energy production in the CNO cycle comes from proton captures followed by a $\beta^{+}$decay, at maximum temperature the rate at which 
energy is produced depends only on the half-lives of the $\beta^{+}$-unstable nuclei and the abundances of the CNO nuclei initially present in the envelope. This is because the CNO reactions do not create new nuclei, but only redistribute them among the various CNO isotopes (Starrfield et al. 1972). Second, the convective region extends throughout the envelope and continuously bring unburned CNO nuclei into the shell source when its temperature is rising very rapidly. This keeps the CNO nuclear reactions operating far from equilibrium. The third effect, mentioned above, is that convection brings both the $\beta^{+}$-unstable nuclei and the energy produced in the shell source to the surface on a rapid time scale. This causes the luminosity to rise to maximum on a convective time scale when the white dwarf radius is still small.

Clearly, an object radiating with luminosity $\sim L_{E d d}$, and $R_{*} \sim 10^{8} \mathrm{~cm}$, will emit copious amounts of EUV and soft X-ray photons. This implies that a nova will be bright in the EUV or soft X-rays for hours or perhaps days before optical maximum. The time scale will depend upon the speed class of the nova. Recent calculations of the peak $T_{e}$ that can be expected under these circumstances demonstrate that temperatures exceeding $10^{6} \mathrm{~K}$ are likely at the time that the $\beta^{+}$-unstable nuclei reach the surface (Starrfield et al. 1989; and these proceedings). They also show that peak $\mathrm{T}_{e}$ is a function of the white dwarf mass (Starrfield et al. 1989).

\section{PHASE II: THE RISE TO VISUAL MAXIMUM}

During the rising branch of the optical light curve, the nova exhibits spectral features corresponding to an optically thick, expanding shell. As the expansion is rapid and the bolometric luminosity is nearly constant, the effective temperature smoothly declines and reaches a minimum of $4 \times 10^{3} \mathrm{~K}$ to $7 \times 10^{3} \mathrm{~K}$ at visual maximum (Gallagher and Starrfield 1978). The maximum in optical light is determined by the temperature and density at which hydrogen recombines and the opacity drops. After this time, a pseudo-photosphere moves inward in mass (Wehrse et al. 1989; these proceedings).

An important advance in the understanding of UV spectra of novae occurred as a direct result of IUE observations of the outburst of SN 1987A. It was realized that the first IUE spectra of SN 1987A strongly resembled UV spectra of novae taken near maximum, except that the lines were much broader in the supernova (Stryker et al. 1988). By using the same techniques that have recently been developed to treat the expanding atmospheres of supernovae, one can analyze the early UV spectra of a nova (Wehrse et al. 1989; these proceedings). These authors report that the spherical, expanding, stellar atmospheres that they have constructed for novae are characterized by a very slow increase of density with decreasing radius. This variation leads to very large geometrical extensions and large temperature differences between the inner and outer layers. The theoretical spectra show a large IR excess and a small Balmer jump which may be either in emission or absorption.

These studies are very important because they provide a completely new method for analyzing the physical conditions in novae during the early stages of the outburst. Once these conditions are known, then it becomes possible to determine the abundances for a variety of species. The abundances derived from spectral line synthesis, using these atmospheres, can then be compared to the nebular analysis methods that are applied 
much later in the evolution of the nova spectrum. These two abundance methods are completely independent and this comparison should ultimately provide a much better determination of the abundances in novae ejecta.

The early spectra obtained in the optical are usually dominated by broad absorption lines and emission lines are either weak or absent. Spectral types are reported to be B to A, although some novae have been observed to have a later spectral class; for example, RR Pic was listed as having a class of $F$ and Nova Vul 1987 was probably cooler. The optical spectrum of V1500 Cyg, one day before maximum, was that of a B2Ia star with unusual absorption line strengths for the CNO elements (Boyarchuk et al. 1977). The analysis of spectra obtained later in the outburst showed that the anomalous strengths were caused by abundance enhancements (Ferland and Shields 1978a; Lance, McCall, and Uomoto 1988). Interestingly enough, the unusual line strengths seen in V1500 Cyg are rather common in novae (McLaughlin 1960).

IR observations, if obtained early in the outburst, show that the nova is radiating like a black body expanding with time. Gallagher and Ney (1976) used this phase to determine the distance to V1500 Cygni. They measured the rate of angular expansion of the photosphere and combined it with the doppler velocities of the lines observed at that time. Given both that the measured velocities correspond to the material that is producing the black body emission and that this material was ejected with spherical symmetry, one can determine a very accurate distance. This black- body expansion parallax method has now been applied to all novae that were observed early enough in the outburst to detect the expanding photosphere (see Gehrz 88; and these proceedings). If a nova forms an optically thick dust shell, then this same method can be applied to the expanding dust shell and provides a second distance estimate for the same nova.

At some time after the expanding gas begins to go optically thin at IR wavelengths, the nova enters the free-free expansion phase (Gallagher and Starrfield 1978). The IR investigators (see Gehrz 1988 and references therein) have shown that the density of the expanding shell can be determined from the wavelength where the optically thin free-free emission turns over into the Rayleigh-Jeans tail. This result is well known from radio studies (Hjellming 1989; these proceedings).

\section{PHASE III: CONSTANT BOLOMETRIC LUMINOSITY}

It is only during the brief period around maximum that pure absorption line or PCygni profiles are seen and are potentially accessible to analysis (Williams et al. 1981). The features that are seen evolve continuously in velocity as the receding photosphere moves inward in mass and many of the absorption lines seen at maximum can eventually be identified with the emission lines in the nebular shell (McLaughlin 1960; Gallagher and Starrfield 1978). Optical observations show P-Cygni profiles in the lines of hydrogen, Fe II, and singly ionized metals. As the nova begins its decline, the continuum fades (it is shifting into the blue) more rapidly then the emission lines and the P-Cygni profiles disappear. After a short time, emission lines dominate the spectrum. They have rather complex profiles and suggest that material has been ejected in clouds, blobs, rings, or jets. The duration of the photospheric phase depends upon the speed class of the nova. In fast, luminous, novae the primary shell may become optically thin in a few days, 
in a slower, lower optical luminosity nova, continuous mass loss can maintain a low temperature photosphere for several months or years.

The hydrodynamic simulations predicted that a phase of constant luminosity would follow the initial outburst (Starrfield et al. 1972; Starrfield, Sparks, and Truran 1974). The cause of this phase is that only a fraction of the accreted envelope is ejected by the initial explosion. The remaining material (anywhere from 10\% to 90\%) quickly returns to quasistatic equilibrium but the envelope now has a radius extending to $\sim 10^{11} \mathrm{~cm}$. Because the shell source is still burning at the bottom of the envelope, $\mathrm{L} \sim \mathrm{L}_{E d d}$ and, therefore, $T_{e}$ will exceed $10^{5} \mathrm{~K}$. It has now been shown that this material can be ejected by radiation pressure driven mass loss (Starrfield et al. 1989). The energy source for the wind can be either dynamical friction during the common-envelope phase (MacDonald 1980; MacDonald, Fujimoto, and Truran 1985) or thermonuclear burning at the bottom of the accreted layer, once the outer envelope of the white dwarf shrinks within its Roche lobe. Nevertheless, as long as some material is still present on the white dwarf, the remnant will radiate at a constant luminosity. X-ray, UV, IR, and optical studies confirm that novae exhibit a constant luminosity phase (Starrfield 1988). The radiated energy, the effective temperature, and the time scale of this phase of the outburst provide fundamental data about the white dwarf (MacDonald, Fujimoto, and Truran 1985; Starrfield et al. 1989). These data have been used to show that the masses of white dwarfs in nova systems range from $\sim 0.6 \mathrm{M}_{\odot}$ to $\sim 1.2 \mathrm{M}_{\odot}$ or higher.

The early post-maximum decline in the optical is the result of the redistribution of the luminosity into the UV as the shrinking pseudo-photosphere moves inward in mass. The stable luminosity is determined by the core mass and can be found from the core mass- luminosity relationship (Paczynski 1971). This relationship gives a value of $\mathrm{M}_{B o l} \sim-7$ for the typical nova which has a white dwarf with a mass exceeding $1.0 \mathrm{M}_{\odot}$. While the bolometric luminosities of the fastest, brightest novae are initially higher than this value, their luminosity drops to this value soon after maximum. In contrast, novae with maximum absolute visual magnitudes fainter than -7 show little change in bolometric luminosity after maximum; the initial decline in the visual can be completely attributed to flux redistribution into the UV and EUV (Starrfield 1988; Snijders et al. 1987; Krautter and Williams 1989).

During the declining branch of the light curve, novae undergo important changes in physical conditions. Because the hardening of the radiation field from the central source produces an ionization front moving out through the expanding ejecta, because the density of matter is high compared to most planetary nebulae, and because the process is time dependent; the spectrum can become exceedingly complex, especially for novae with significant post-maximum stellar winds (Gallagher and Starrfield 1978; Friedjung 1989; these proceedings). The most important change in optical and UV spectra is the rapid growth of both permitted and forbidden emission lines.

The emission lines contain a wealth of information about the conditions in the ejected envelope and the nature of the central star. The basic trend, as the nova declines, is for lines requiring lower electron densities and higher electron temperatures to gradually dominate the spectrum (Gallagher and Starrfield 1978). The cause is obvious: the shell is expanding and the effective temperature of the central source is rising to values exceeding $\sim 3 \times 10^{5} \mathrm{~K}$ (Gallagher and Starrfield 1976; Lance, McCall, 
and Uomoto 1988; Ögelman, Krautter, and Beuermann 1987; Krautter and Williams 1989; Starrfield et al. 1989). In addition to the optical forbidden lines commonly seen in novae and planetary nebulae such as [OII] $3727 \AA$, [OIII] $4363 \AA$, [NIII] $4640 \AA$, [OIII] $4959 \AA$, and [OIII] $5007 \AA$; some novae are also observed to show coronal lines such as [Fe VII] $6087 \AA,[\mathrm{FeX}] 6374 \AA$, and [FeXI] $7892 \AA$. GQ Mus still exhibits coronal line emission more than four years after the initial explosion and, currently, $[\mathrm{FeX}]$ is stronger than $\mathrm{H} \alpha$ (Krautter and Williams 1989). Other novae have been found to display IR coronal line emission (Gehrz 1989; preprint) and analyses of these data indicate that these novae have ejecta enriched in the intermediate mass elements. Photoionization from a hot central source may be sufficient to explain the presence of these lines very late in the outburst (Krautter and Williams 1989).

Forbidden line emission from a source as complex as a nova in outburst provides great difficulty in both analysis and interpretation (Ferland and Shields 1978a; Lance, McCall, and Uomoto 1988). The problems involve departures from spherical symmetry and inhomogeneities in the expanding material, but become more tractable at late stages in the outburst when the ejecta are completely ionized and recombinations rather than collisions dominate. The analyses of emission lines in the nebular stage currently provide the most accurate abundance determinations for novae. These analyses are based upon standard techniques developed to study planetary nebulae (Williams et al. 1981).

As in planetary nebulae, one can solve for the electron density and temperature by simultaneously solving a system of equations involving various line ratios as a function of time. The most useful in novae are the ratios: [OIII] $(4959 \AA+5007 \AA) /$ [OIII] $(4363 \AA) ;[\mathrm{OIII}](4959 \AA+5007 \AA) /[\mathrm{NeIII}](3869 \AA)$; and [OIII] $(4959 \AA+5007 \AA) / \mathrm{HeI}$ $(5876 \AA)$. These lines are generally strong and can be measured with high signal to noise. When applied over the time of the outburst, they are constrained by the fact that the elemental abundances have to be constant. The excitation of the emission lines increases steadily with time and it is not uncommon to find lines of triply or quadruply ionized elements in either the optical or UV spectra (Gallagher and Starrfield 1978). In the last stages of the optical outburst the ionization increases to levels of 50-60 ev and the electron densities decrease to values of $10^{8}$ to $10^{10} \mathrm{~cm}^{-3}$. A detailed discussion of V1500 Cyg and references to some of the necessary constants can be found in Lance, McCall, and Uomoto (1988). V1370 Aql and V693 CrA have also been observed and analyzed in great detail (Snijders et al. 1987; Williams et al. 1985). They found a large range in ionization potential of the emission lines which suggested that the gas in these novae was clumpy and the physical conditions varied from one clump to another. The abundance distributions found for each nova, by these two different groups, indicated that the explosion occurred on an $\mathrm{ONeMg}$ white dwarf in both systems.

At later stages in the decline from optical maximum, new absorption line systems appear at higher velocities, compared to the principal absorption line system. These systems are called "diffuse enhanced" and consist of broad features that may separate (as time passes) into many sharp subcomponents. At still later times in many novae, very highly ionized features appear in absorption at even larger velocities than the diffuse enhanced systems. These systems are collectively called the "Orion" spectrum and are optical analogues of the "sharp, narrow, absorption" components seen in the UV spectra of the most luminous $O$ and B stars that exhibit strong stellar winds. 
During the decline from optical maximum, some two to three months into the outburst, most novae begin to develop an IR excess. This excess is caused by the formation and growth of grains in the expanding shell which then reradiate the UV energy from the hot, luminous, white dwarf. A comprehensive review of the IR studies of novae in outburst can be found in Gehrz (1988; see also Gehrz 1989, these proceedings). The key fact about the IR studies is that they are the only means, at present, of detecting and studying the formation and evolution of dust in the ejecta.

When first detected, the IR excess attributed to grains exhibits a continuum black body temperature slightly exceeding $10^{3} \mathrm{~K}$. If this material has just formed, then this temperature is considerably below a value of $\sim 2 \times 10^{3} \mathrm{~K}$ at which the grains are normally expected to form (Gehrz 88). As time passes, the grain emission slowly decreases in temperature to values around $8 \times 10^{2} \mathrm{~K}$. Gehrz (1988) explains the variation in temperature as the formation of small particles which are inefficient radiators. They slowly grow to a size that exceeds that of normal interstellar particles $(0.01 \mu \mathrm{m}-0.03 \mu \mathrm{m})$. The composition of the grains was thought to be some form of amorphous carbon since, until recently, the IR excess failed to show any features due to silicates (Gehrz 88). This picture has now changed completely. IR observations of V1370 Aql showed that it exhibited an excess with a broad continuum peaked at about $8 \mu \mathrm{m}$ and superimposed on this broad excess was a feature at $10 \mu \mathrm{m}$ that has yet to be identified. This nova was later discovered to be unusual because it ejected material with a very strange abundance distribution (Snijders et al. 1987). After this result, QU Vul was observed to form silicate grains since its IR emission showed both the $10 \mu \mathrm{m}$ and the $20 \mu \mathrm{m}$ features seen in other classes of astronomical objects (Gehrz 1988). However, it showed no continuum excess and its IR emission never became optically thick (Gehrz et al. 1986). Our understanding of grain formation in novae has now become even more cloudy since detailed studies of Nova Vul 1987 (Gehrz 1989; these proceedings and private communication), using the Wyoming Infrared Observatory, have made positive identification of four different kinds of dust: amorphous carbon, silicon carbide, silicates, and PAH. The determination of how a single outburst could provide the materials necessary to form all of these kinds of dust will prove difficult.

This leads to an interesting puzzle about the IR excesses of novae: some novae show optically thick emission from grains while others exhibit only optically thin emission. In both cases they develop IR emission, characteristic of grain formation, but in the optically thin case the amount of energy radiated by the grains never approaches the values emitted by the nova at maximum optical light. In contrast, in the optically thick case, the IR emission in the nova reaches the same amount of energy as was observed early in the outburst. In addition, some of the optically thin emitting novae will show no indication, whatsoever, in their optical light curve that dust has formed in the ejecta. A possible explanation for this phenomenon is that the region where the dust is forming has been ejected asymmetrically and does not completely block the UV light from the central source (Gehrz 88).

One nova that did not show an excess until very late in its outburst was V1500 Cygni. It was proposed that the excess was caused not by grains but by strong emission from [NeII] at $12.8 \mu \mathrm{m}$ (Ferland and Shields 1978b). The presence of this line in novae was finally confirmed in QU Vul and was instrumental in showing that neon was 
overabundant in this nova (Gehrz et al. 1986; Starrfield, Sparks, and Truran 1986).

Radio studies of novae are reviewed by Hjellming (1989; these proceedings) who has taken part in most of the studies that have been done on novae. It is an excellent review and I will not repeat any of it here.

\section{PHASE IV: THE RETURN TO QUIESCENCE}

One remaining question about the nova outburst concerns the time scale of the constant luminosity phase; i.e., how long does it take for the cessation of nuclear burning and the return of the nova to its quiescent state. Observations show that this phase proceeds more rapidly than the nuclear burning time scale for the remnant hydrogen envelope would predict. This implies that some mechanism (or mechanisms) must be at work to remove the remnant envelope and terminate the outburst. Whatever this mechanism is, as the mass of the remnant envelope decreases with time, we can expect the radius of the remnant to shrink and the size of the star will return to that expected for an equilibrium white dwarf. This phase, however, will occur at nearly constant luminosity and so the effective temperature will increase. As shown by the observations of those novae that show coronal lines, and especially by those of GQ Mus (Krautter and Williams 1989), $\mathrm{T}_{e}$ can reach values exceeding $5 \times 10^{5} \mathrm{~K}$. Discussions of the mechanisms and time scales for turn-off can be found in Starrfield (1979), Truran (1982), MacDonald, Fujimoto, and Truran (1975), and Starrfield et al. (1989).

As mentioned in the last section, I assume that radiation pressure is driving mass off the remnant at a rate that can be calculated by expressions provided by Castor, Abbot, and Klein (1975; see also Abbot 1982; and references therein). I assume that the energy source for the wind is dynamical friction during the common- envelope phase of this evolution, for as long as the radius of the remnant overfills the Roche Lobe of the white dwarf, and thermonuclear burning in the later stages when the radius of the remnant is less than that of the Roche Lobe. Actually thermonuclear burning is important at all stages of the post-explosion evolution but this argument does not depend on the actual mechanism. I also assume that the white dwarf is radiating at the luminosity given by the core mass-luminosity relationship (Paczynski 1971). It is then possible to calculate the time scale for the white dwarf to eject its entire envelope and this turns out to be a strong function of white dwarf mass. Starrfield et al. (1989) show that the time scale for ridding the envelope of the accreted mass is less than a year for novae with the most massive white dwarfs such as U Sco and V394 CrA. In contrast, the time scale for a $1.0 \mathrm{M}_{\odot}$ white dwarf is more than 100 years. They also show that radiation pressure driven mass loss is probably responsible for removing all of the accreted mass left on the white dwarf and returning it to minimum. The nova will evolve at a constant luminosity, but steadily increasing $\mathrm{T}_{c}$, until the amount of mass remaining on the star is too small to support further nuclear burning. At this time the remnant material, if any, will collapse back onto the surface of the star on a time scale of weeks. Near the end of the outburst the nova will go through a phase where the temperature approaches values of $10^{6} \mathrm{~K}$ or higher. Therefore, the most interesting wavelength regime to study a nova at this time is the $\mathrm{X}$-ray.

EXOSAT observations of GQ Mus, PW Vul, QU Vul, and RS Oph (Mason et al. 1986; Ögelman, Krautter, and Beuermann 1987) implied that the central source, at the 
time the observations were made, was radiating at $\mathrm{L} \sim L_{E d d}$ with an effective temperature of $\sim 3 \times 10^{5} \mathrm{~K}$. However, the calculations of Starrfield, Sparks, and Truran (1985) and MacDonald, Fujimoto, and Truran (1985) show that the effective temperature should reach much higher values than found in the $\mathrm{X}$-ray observations. The temperatures found above are more consistent with low mass (large radius) white dwarfs than with the high mass white dwarfs predicted by the thermonuclear runaway theory (Starrfield, Truran, and Sparks 1978; Starrfield 1979). Note, however, that recent optical spectra suggest that the central source has continued to increase in temperature (Krautter and Williams 1989). Both GQ Mus and QU Vul have been studied with the IUE satellite at a very late stage in their outburst (Krautter and Starrfield 1989; in preparation). An SWP spectrum was obtained for each object in September 1989 and each spectrum shows a continuum with superposed emission lines. It appears that neither of these novae have turned off.

\section{SUMMARY AND DISCUSSION}

In this review I have tried to show the importance of multi-wavelength observations of novae in outburst. Observations at a variety of wavelengths throughout the evolution of the nova have provided important information about the characteristics of the explosion and the long term evolution of the system. Every time a new wavelength regime has been applied to studies of the nova outburst, new and important information has been learned about the characteristics of the outburst.

The X-ray studies of EXOSAT have determined the temperature of the central source at late times in the outburst. The observed temperatures of $3 \times 10^{5} \mathrm{~K}$ demonstrate that a hot, luminous white dwarf must exist within the system. However, this temperature is lower than recent predictions of the temperatures that should be attained in the final evolution of a nova to quiescence. On the other hand, recent optical studies have found intense coronal line emission from some of the same novae observed by EXOSAT. For example, GQ Mus currently shows [Fe X] stronger than $\mathrm{H} \alpha$ indicating a hot white dwarf with a temperature exceeding $10^{6} \mathrm{~K}$. This value is in good agreement with the predictions for massive white dwarfs.

The UV studies have proved to be very valuable for determining the elemental abundances in the ejected material. This is because there are lines in the UV that come from species that do not emit in the optical. As a direct result of spectra obtained with the IUE satellite, we have identified a class of novae that are occurring on $\mathrm{ONeMg}$ white dwarfs. It is now possible to determine elemental abundances for novae at early stages, using IUE spectra and spherical, expanding, stellar atmospheres. Comparison of this method with standard nebular analyses techniques, done for the same nova will, undoubtedly, provide important data both about novae and the methods themselves.

One can use the IR emission at two different phases, the early black-body expansion phase and the early part of the dust condensation phase, to determine an expansion parallax to the nova. This is the most accurate early distance method. At late times the direct expansion of the shell can usually be measured (Cohen 1985). In addition, the IR observations are the only method in which we can study the formation and evolution of grains in the ejected shell. The grain studies show that a variety of species can be 
formed in novae ejecta such as amorphous carbon, silicates, and PAH. A most exciting recent discovery has been that Nova Vul 1987 formed at least four different kinds of grains.

Optical studies have been done for novae for many years and they have provided fundamental data on the shapes and distribution of the mass ejected during the outburst, on the elemental abundances for those elements that have lines in the optical region of the spectrum, and on the polarization.

Data obtained in the radio provides important information about the space distribution of the gas ejected during the outburst. For example, at least one nova has been shown to have ejected material in a jet (Hjellming 1989; these proceedings). Radio data also provides independent information about the masses, temperatures, and velocities of the ejected material. It is also possible to determine the angular sizes as a function of time by frequent imaging of nova shells.

I would like to express my thanks for many useful discussion to Drs. R. Gehrz, R. Hjellming, J. Krautter, G. S. Kutter, S. Shore, E. M. Sion, W. M. Sparks, G. Sonneborn, J. Truran, R. Wade, R. M. Wagner, and R. E. Williams. I am also grateful to Drs. S. Colgate, A. N. Cox, C. F. Keller, M. Henderson, and K. Meyer for the hospitality of the Los Alamos National Laboratory and a generous allotment of computer time. This work was supported in part by NSF Grants AST85-16173 and AST88-18215 to Arizona State University, by the Institute of Geophysics and Planetary Physics at Los Alamos, by NASA grants to Arizona State University and to the University of Colorado, and by the DOE.

\section{REFERENCES}

Abbott, D. C. 1982, Ap. J., 259, 282.

Boyarchuk, A.A., Galkina, T.S., Krasnobabtsev, V.I., Rachkovskaya, T.M., and Shakhovskaya, N.I. 1977, Sov. A. J., 21, 257.

Castor, J. I., Abbott, D. C., and Klein, R. I. 1975, Ap. J., 195, 157.

Cohen, J. G. 1985, Ap. J., 292, 90. Ferland, G.J. and Shields, G.A. 1978a, Ap. J., $226,172$.

Ferland. G.J., and Shields, G.A. 1978b, Ap. J. (Letters), 224, L15.

Gallagher, J.S., and Ney, E.P. 1976, Ap. J. (Letters), 204, L35.

Gallagher, J.S. and Starrfield, S.G. 1976, M.N.R.A.S., 176, 53.

Gallagher, J.S. and Starrfield, S.G. 1978, Ann. Rev. Astr. and Ap., 16, 171.

Gehrz, R. D. 1988, Ann. Rev. Astr. and Ap., 26, 377.

Gehrz, R.D., Grasdalen, G.L., Greenhouse, M., Hackwell, J.A., Hayward,T., and Bentley, A.F. 1986, Ap. J. (Letters), 308, L63.

Krautter, J., and Williams, R. E. 1989, Astrophys. J., 341, p. 968.

Lance, C.M., McCall, M.M., and Uomoto A. K. 1988, Ap. J. Supp., 66, 151.

MacDonald, J. 1980, M.N.R.A.S., 191, 933.

MacDonald, J., Fujimoto, M. Y., and Truran J. W. 1985, Ap. J., 294, 263.

Mason, K.O., Córdova, F. A., Bode, M. F., and Barr, P. 1986, in RS Oph and the Recurrent Nova Phenomenon, ed. M. F. Bode (VNU Press; Utrecht), p 167. 
McLaughlin, D. B. 1960, in Stellar Atmospheres: Stars and Stellar Systems VI, ed. J.

S. Greenstein (Chicago :University of Chicago Press), p. 585.

Ögelman, H., Krautter, J., and Beuermann, K. 1987, Astr. Ap., 177, 110.

Paczynski, B. 1971, Acta Astron., 21, 271.

Shara, M. M. 1989, Public. Astron. Soc. Pac., 101, p. 5.

Snijders, M.A.J., Batt, T.J., Roche, P. F., Seaton, M. J., Morton, D. C., Spoelstra, T.A.T., and Blades, J. C. 1987, M.N.R.A.S., 228, 329.

Sparks, W.M., Starrfield, S., Truran, J. W., and Kutter, G. S. 1988, in Atmospheric Phenomena in Stellar Explosions, ed. K. Nomoto, (Springer-Verlag: Heidelberg), p. 234.

Starffield, S. 1979, in White Dwarfs and Variable Degenerate Stars, ed. H.M. Van Horn and V. Weidemann (Rochester: University of Rochester), p. 274.

Starrfield, S. 1986, in Radiation Hydrodynamics, ed. D. Mihalas, and K.-H. Winkler, (Dordrecht: Reidel), p. 225.

Starrfield, S. 1988, in Multiwavelength Astrophysics, ed. F A. Córdova, (Cambridge: University Press), p. 159.

Starrfield, S. 1989, in The Classical Nova, ed. N. Evans, and M. Bode, (New York: Wiley), p. 39.

Starrfield, S., Sparks, W. M., and Truran, J. W. 1985 Ap. J., 291, 136.

Starrfield, S., Sparks, W. M., and Truran, J. W. 1986, Ap. J. (Letters), 303, L5.

Starrfield, S., Sparks, W. M. and Truran, J. W. 1974, Ap. J. Suppl., 28, 247.

Starrfield, S., Truran, J. W. and Sparks, W. M. 1978, Ap. J., 226, 186.

Starrfield, S., Truran, J. W., Sparks, W. M., and Krautter, J. 1989, in The Future of EUV Astronomy, ed. R. Malina, in press.

Starrfield, S., Truran, J.W., Sparks, W.M. and Kutter, G. S. 1972, Ap. J., 176, 169.

Stryker, L. L., Hestand, J., Starrfield, S., Wehrse, R., and Shaviv, G. 1988, in A Decade of UV Astronomy with the IUE Satellite, ed. E. Rolfe, (Noordwijk: Estec Publications: SP- 263), p. 149.

Truran, J. W. 1982, in Essays in Nuclear Astrophysics, ed. C.A. Barnes, D.D. Clayton, and D. Schramm (Cambridge: Cambridge U. Press), p. 467.

Truran, J. W., and Livio, M. 1986, Ap. J., 308, 721.

Williams, R.E., Sparks, W.M., Gallagher, J.S., Ney, E.P., Starrfield, S., and Truran, J. W. 1981, Ap. J., 251, 221.

Williams, R.E., Sparks, W.M., Ney, E.P., Starrfield, S., and Truran, J. W. 1985, Mon. Not. Roy. Astron. Soc., 212, 753. 